\title{
Closed-loop atmospheric dispersion compensation: on-sky demonstration of sub-milliarcsecond residual dispersion across H-band
}

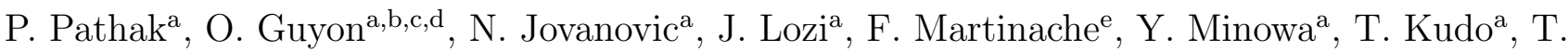 \\ Kotani $^{\mathrm{f}}$, and H. Takami ${ }^{\mathrm{f}}$ \\ ${ }^{a}$ National Astronomical Observatory of Japan, Subaru Telescope, National Institutes of \\ Natural Sciences, Hilo, HI, 96720, U.S.A. \\ ${ }^{\mathrm{b}}$ Astrobiology Center, 2-21-1 Osawa, Mitaka, Tokyo, 181-8588, Japan \\ ${ }^{\mathrm{c}}$ Steward Observatory, University of Arizona, Tucson, AZ, 85721, U.S.A. \\ ${ }^{\mathrm{d} C o l l e g e}$ of Optical Sciences, University of Arizona, Tucson, AZ 85721, USA \\ e Observatoire de la Cote d'Azur, Boulevard de l'Observatoire, Nice, 06304, France \\ ${ }^{\mathrm{f}}$ National Astronomical Observatory of Japan, 2-21-1 Osawa, Mitaka, Japan
}

\begin{abstract}
On ground-based telescopes employing adaptive optics (AO) systems, atmospheric dispersion compensation is essential to deliver high quality imaging, and critical for coronagraphy and precision astrometry. In AO systems delivering high-Strehl, residual dispersion if often a dominant source of error, and is especially challenging to correct on large aperture telescopes. Imperfect compensation by atmospheric dispersion compensator (ADC) can be due to errors in the atmospheric dispersion estimation (usually derived from local temperature and pressure measurements), or calibration errors in the instrument and ADC optics. These limitations can be addressed with a high-precision on-sky measurement of residual dispersion in a closed-loop control scheme. In this work, we present a focal plane based technique, which utilizes the chromatic scaling of speckles to measure residual dispersion (atmospheric and optical) in the final science image. By using an adaptive speckle grid generated using a deformable mirror (DM) with a sufficiently large number of actuators, the residual dispersion is accurately measured and subsequently corrected in a closed-loop control. We demonstrate on-sky residual dispersion of 0.75 mas across H-band, reduced from 8.4 mas before closing the loop. This work will be useful for upcoming extremely large telescopes (ELTs) to achieve sub-milliarcsecond precision on astrometry and residual dispersion.
\end{abstract}

Keywords: Adaptive Optics, Atmospheric dispersion, ADC, Exoplanets

\section{INTRODUCTION}

Traditionally, atmospheric dispersion is compensated for by an atmospheric dispersion compensator (ADC). ${ }^{1,2}$ The ADC control relies on an a priori model of the atmosphere whose parameters are solely based on the pointing of the telescope (the model often also includes temperature and pressure as an input), which is too simplistic and results in an imperfect compensation, leading to some residuals. The presence of residual atmospheric dispersion (chromaticity) becomes a dominant source of noise once other wavefront errors have been accounted for, specially in the Extreme AO regime. Chromatic effects will significantly impact the performance of AO on ELTs, especially for high-Strehl ratio performance. For ELTs, the diffraction limited PSF will be $\sim 6-8$ mas at $1 \mu \mathrm{m}$, a tip-tilt error of 1 mas RMS will reduce the Strehl ratio by a factor of $0.82 .^{3}$

Besides affecting the Strehl ratio of the PSF, chromaticity also affects high-performance coronagraphy. ELTs are well suited for imaging habitable exoplanets around M-type stars using the reflected light. This requires a contrast of $10^{-7}$ at $2 \lambda / D$, which can be achieved by employing high-performance - small inner working angle (IWA) - coronagraphs. The contrast at small IWA is limited by stellar angular size and residual low-order

Further author information: (Send correspondence to P. Pathak), E-mail: prashant@naoj.org, Telephone: +01 808 9345081 

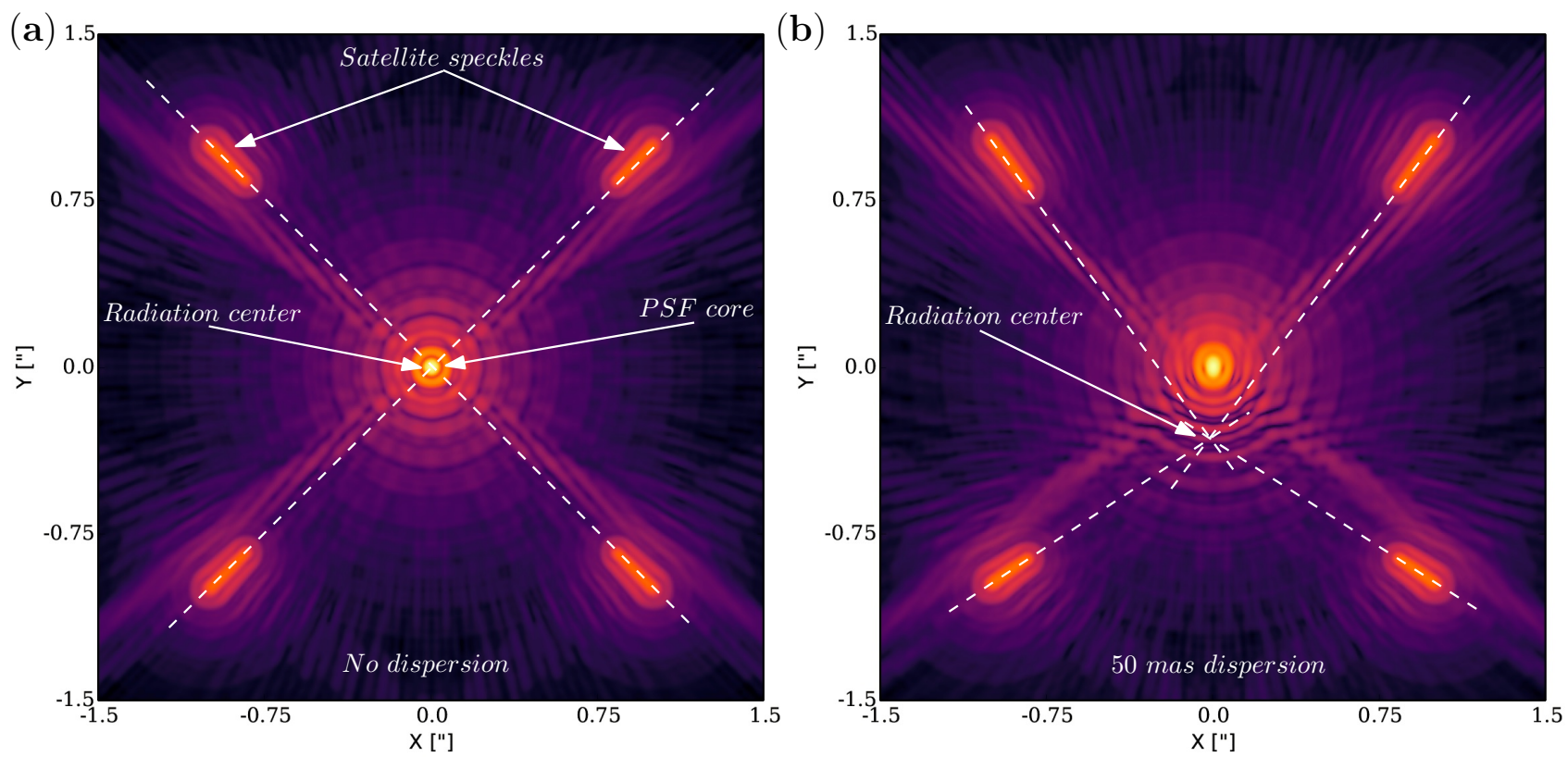

Figure 1. Simulated H-band PSF with artificial satellite speckles: (a) With no atmospheric dispersion, i.e. telescope pointing at zenith, speckles point to the PSF shown by over-plotted lines. (b) PSF with 50 mas atmospheric dispersion in the vertical direction.

aberrations. The angular stellar size is typically around 1 mas, and creates a stable and well-understood residual leak in the focal plane. The aim is then to keep all other sources of leakage - e.g. tip-tilt, low-order modes, residual dispersion - smaller than 1 mas. Atmospheric dispersion creates a small tip-tilt in the science path assuming a perfect correction of tip-tilt in the wavefront sensing path. This can be measured by coronagraphic low order wavefront sensors (LOWFS) and corrected by driving the DM. However the atmospheric dispersion within the science band is not addressed by coronagraphic LOWFS. For ELTs, residual dispersion measurement requirement is 0.1 mas and correction at 0.3 mas level.

The current ADC systems, working based on a look-up table are far from achieving above stipulated requirements. The main drawbacks are no real-time measurement of dispersion and slow temporal response by ADCs, addresses only static component of dispersion (dynamic term is $\approx 0.5$ mas RMS in H-band for $0.6^{\prime \prime}$ seeing). Previously we presented a new focal plane based technique to measure the presence of residual atmospheric dispersion on-sky with a high-precision. ${ }^{4,5}$ In this paper we present closed-loop correction of dispersion and improvement in flux suppression by a high-performance vortex coronagraph by closed-loop correction of dispersion.

\section{MEASURING ATMOSPHERIC DISPERSION}

The concept behind the measurement of dispersion utilizes the chromatic scaling of speckles in the focal plane, explained with the aid of simulation in Figure 1. It features a H-band PSF with conveniently placed symmetric off-axis diffraction features (speckles). The speckles are generated by diffracting the PSF horizontally and vertically in a similar manner to diffraction with or by a grating. Unlike the core of the PSF, off-axis diffraction features are wavelength dependent and appear spectrally dispersed, longer wavelengths diffract more compared to shorter wavelengths. In the case of the PSF with no dispersion (corresponding to a pointing of the telescope at zenith), all the wavelengths are co-focused at the PSF core. The speckles are dispersed radially away from the PSF, pointing towards its location. The location where the speckles seem to radiate from is called the radiation center, which coincides with the PSF core in the absence of dispersion (Fig 1 (a)). The Figure 1 (b) shows how speckles are affected by the presence of dispersion in the PSF. In the presence atmospheric dispersion, the speckles no longer radiate from the PSF core, but from the radiation center, offset from the PSF core. The offset of the radiation center from the PSF core is directly proportional to the presence of dispersion in the PSF. The 


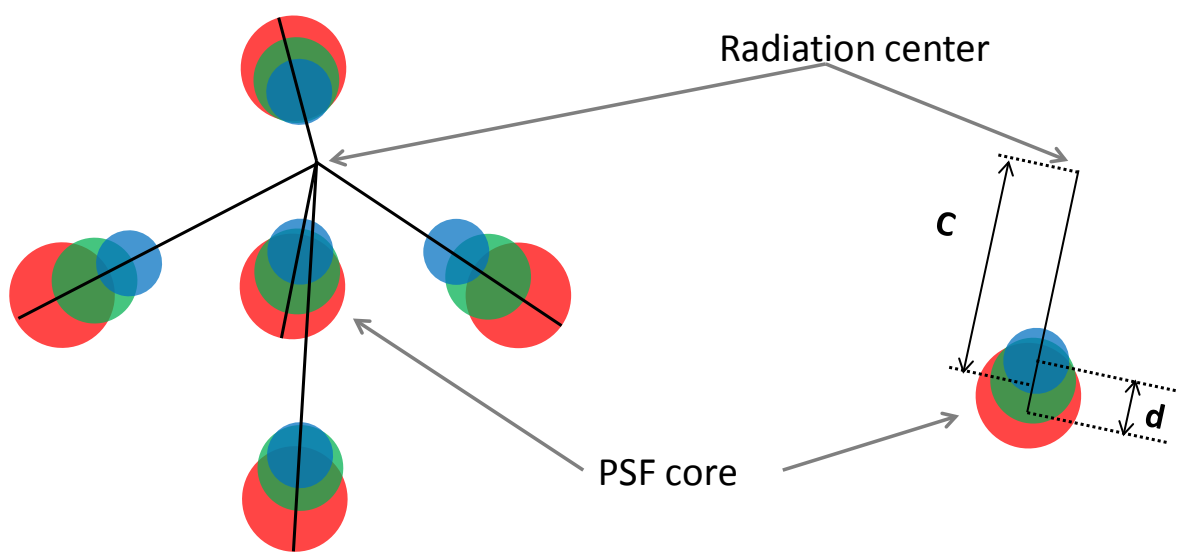

Figure 2. Schematic showing the PSF and satellite speckles in the presence of dispersion. The PSF and satellite speckles radiate (spectrally dispersed) from the radiation center.

presence of dispersion in the PSF can be determined by measuring offset of the radiation center from the PSF core, which is discussed next.

The relationship between the amount of dispersion and deviation of the radiation center from the PSF core can be easily calculated by using the principle of diffraction. Figure 2 shows schematic of the PSF with satellite speckles in the presence of atmospheric dispersion. The dispersion in the PSF is represented by $d$ and offset of the radiation center is given by $C$. The PSF and satellite speckles are spectrally dispersed from the radiation center. The distance of the PSF core and satellite speckles from the radiation center is directly proportional to wavelength $(=$ cont $\times \lambda)$. The ratio between, distance to shortest and longest wavelength of the PSF core can be written as,

$$
\frac{C-\frac{d}{2}}{C+\frac{d}{2}}=\frac{\text { const } \times \lambda_{\min }}{\text { const } \times \lambda_{\max }} .
$$

After rearranging we get,

$$
\begin{aligned}
\frac{d}{C} & =\frac{\left(\lambda_{\max }-\lambda_{\min }\right)}{\frac{\lambda_{\max }+\lambda_{\min }}{2}} \\
& =\frac{\text { Difference between extreme wavelegnths }}{\text { central wavelength }} \text { (within a band) }
\end{aligned}
$$

Using the relationship given by the Eq. 2, the amount of dispersion present in the PSF can be estimated by measuring the distance between the radiation center and the PSF core. The next section, discusses measurement of the radiation center with a high precision.

\subsection{Finding radiation center}

The location of the radiation center was measured by a raster scan around the PSF core to minimize the norm of the difference between original and stretched speckles, the minimum provides a high-precision measurement of the radiation center. The following steps were used for the extraction of the radiation center from an on-sky data, first: the location of the PSF core was found by using the center of gravity method. After finding the PSFs location, it was masked by a square array to remove the PSF core (by setting the pixel values to zero). The image after masking consisted of eight speckles, which were then thresholded to remove the background. The intermediate result of such a process is shown in the Figure 3. The figure shows subtraction of original (speckles in green) and stretched (speckles in red) images (radially stretched copy of the original image), the green colorbar shows counts (positive) for the original image and red colorbar (negative counts) for the subtracted stretched 

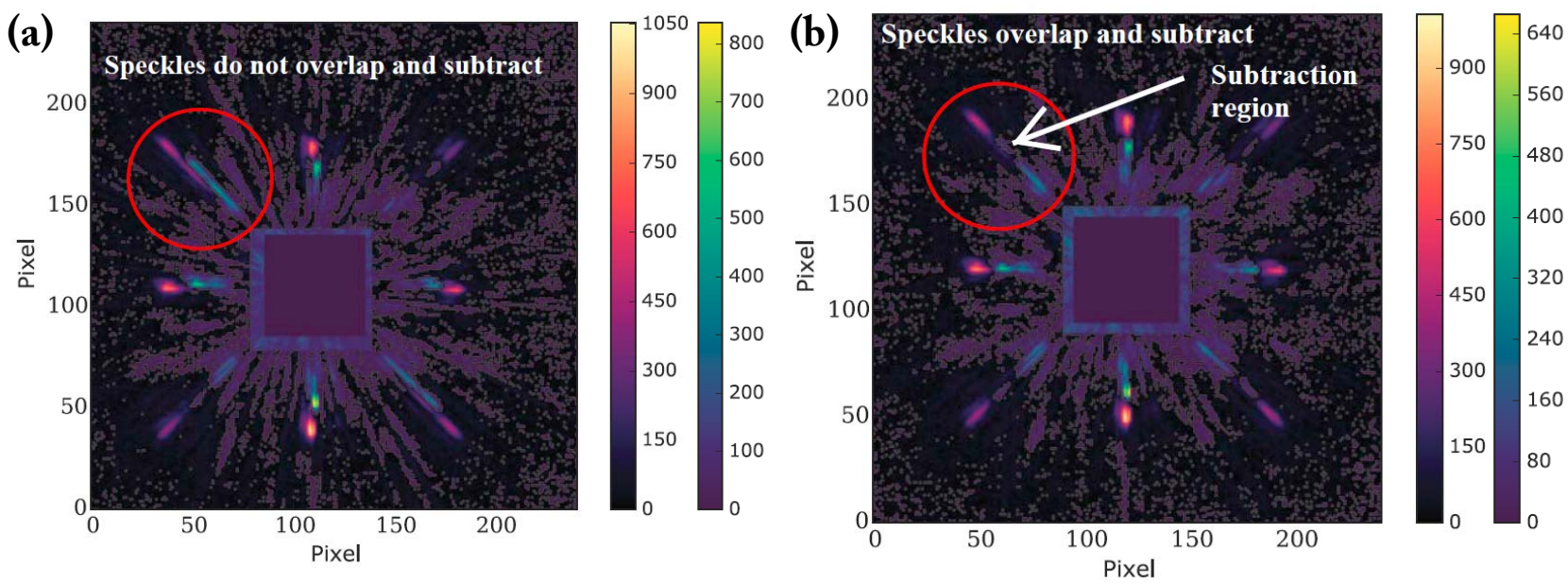

Figure 3. Subtraction of original (green) and stretched (red) images to find the location of the radiation center. (a) Subtraction of images from a pixel coordinate far from the radiation center: original and stretched images do not overlap. The color bar on the left represents negative values, and the one of the right for positive values. (b) Subtraction of images from a pixel coordinate closer to the radiation center: original and stretched images overlap.

image. Figure 3 (a) Shows subtraction of images from pixel coordinate far from the radiation center, original and stretched images do not overlap. Figure 3 (b) represents a case when the stretch and subtraction was from a pixel coordinate closer to the radiation center and it can be seen from the figure (see red circle) original and stretched images overlap and in between the speckles there are regions with close to zero counts. The norm of the difference for Figure 3 (a) will give higher counts of all pixels compared to the Figure 3 (b).

The pseudo code and algorithm to find the location of the radiation center is given as,

$$
\begin{aligned}
& \text { For } i \in-20,20 \\
& \qquad \begin{aligned}
\text { For } & j \in-20,20 \\
& x_{0}, y_{0}=x_{c}+i, y_{c}+j \\
& \mathcal{L}\left(x_{0}, y_{0}\right)=\sum_{x, y}\left|\operatorname{Im}(x, y)-\operatorname{Im}\left(\left(x-x_{0}\right) \alpha+x_{0},\left(y-y_{0}\right) \alpha+y_{0}\right)\right| .
\end{aligned}
\end{aligned}
$$

Where $x_{0}, y_{0}$ are coordinate of stretch, which are centered around the PSF core position $\left(x_{c}, y_{c}\right), \alpha$ is the amount of stretch, $i$ and $j$ represent range of raster scan around the PSF core. For practical purpose the value of $\alpha$ used was $1.2(20 \%)$. We have established, how to measure the location of the radiation center and calculation of dispersion from it, next we present on-sky results. For detailed explanation behind the measurement and correction of the dispersion see Refs. 4.

\section{ON-SKY RESULTS}

\subsection{Experimental setup}

The measurement of dispersion utilizes modules inside Subaru Coronagraphic Extreme Adaptive Optics (SCExAO) ${ }^{6}$ and correction is done by driving the science path ADC inside AO188. ${ }^{7,8}$ As shown in Figure 4, SCExAO sits behind the facility AO instrument AO188 and utilizes partial AO correction. In closed loop, AO188 offers Strehl ratios in the H-band between $20 \%-40 \%$ and SCExAO improves it to $70 \%-90 \%$. The calibration speckles are generated by the DM of SCExAO as shown in Figure 4 and images (y-H band) are acquired using internal NIR camera $(320 \times 256$ pix InGaAs detector). The measurement of dispersion is done using the concepts explained previously. Once the presence of dispersion is calculated, correction offsets are sent to the ADC prisms of AO188.

For closed-loop correction of dispersion, the AO correction from AO188 was only used and ExAO performance of SCExAO was not utilized. At the time of the test due to limitations in the SCExAO control software, ExAO loop and satellite speckles could not be deployed simultaneously. 


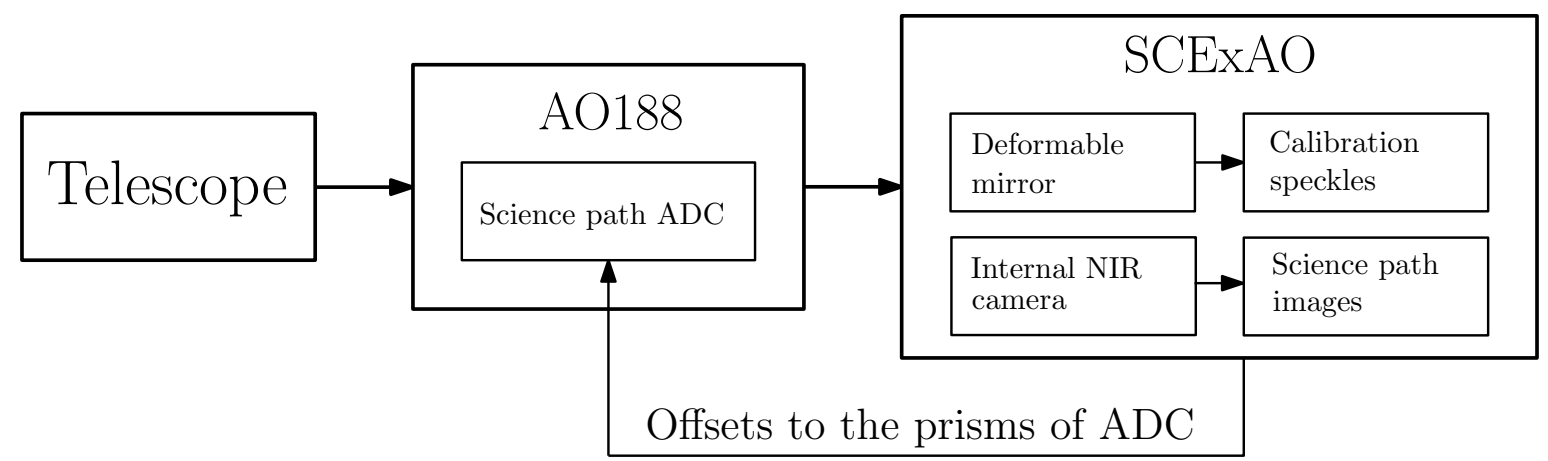

Figure 4. Schematic showing the modules behind the measurement and correction of dispersion.

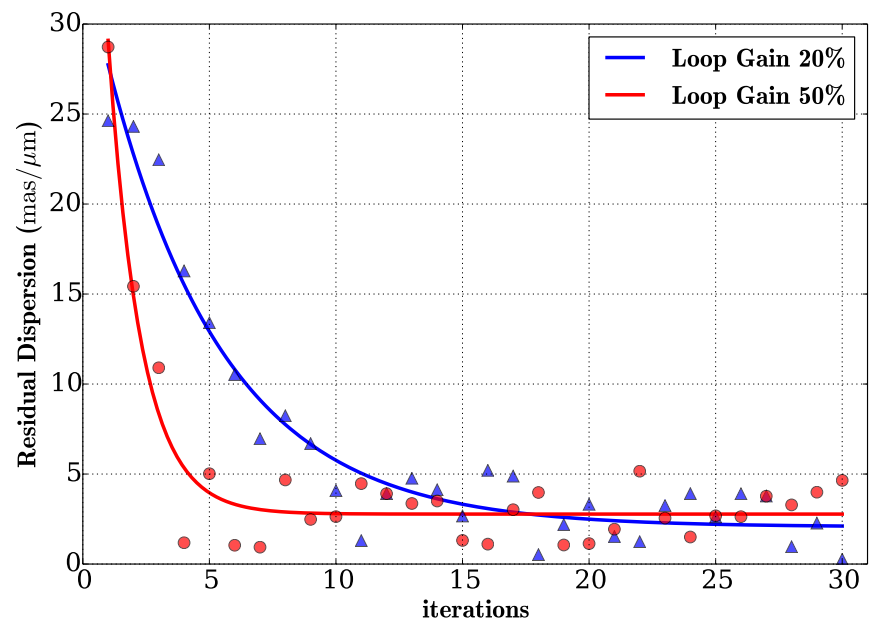

Figure 5. On-sky closed-loop correction of residual dispersion for two different loop gains. For larger loop gain, loop converges faster compared to smaller gain.

\subsection{Closed-loop correction}

The on-sky closed-loop correction of residual atmospheric dispersion was achieved on the target Beta Andromedae (spectral type M0, R-mag $=0.81, \mathrm{H}-\mathrm{mag}=-1.65$ ) on one of the SCExAO's engineering run on September $19^{t h}$, 2016. For closed-loop test an average of 20 measurements was used to average the dispersion due to atmospheric tip-tilt. Figure 5 shows magnitude of residual dispersion (post look-up table based correction) as function of iterations. The closed-loop measurement and correction was performed for two loop gains of $20 \%$ and $50 \%$, the loop converges faster for a loop gain of $50 \%$. The data points are fitted with exponential function of the form, $y=a \times \exp (-b \times x)+c$. The value of residual dispersion went from 24 mas $/ \mu \mathrm{m}$ to $2.5 \mathrm{mas} / \mu \mathrm{m}$, which corresponds to PSF elongation of 7.2 mas to 0.75 mas in H-band before and after correction respectively.

In this section, improvement in the accuracy by averaging the measurements is discussed. Figure 6 shows 1000 measurements of residual dispersion before and after closing the loop, shown using green and red symbols respectively. The measurements were binned into 100 bins, each bin contains an average of 10 measurements. The binned measurements are shown by blue and cyan symbols before and after closing the loop respectively. The measurement accuracy went up by averaging the measurements. The factors affecting the measurement can be read noise of the camera and dispersion due to atmospheric til-tilt. This shows, to correct for the static component of the dispersion (due to elevation of the telescope) it is important to average the measured residuals.

\subsection{Effect of residual dispersion on a high-performance Vortex coronagraph}

The Vector Vortex Coronagraph (VVC) is a phase-masked coronagraph, one of the most efficient in terms of IWA and throughput. The working principle behind VVC is a continuous helical phase ramp in the focal plane 


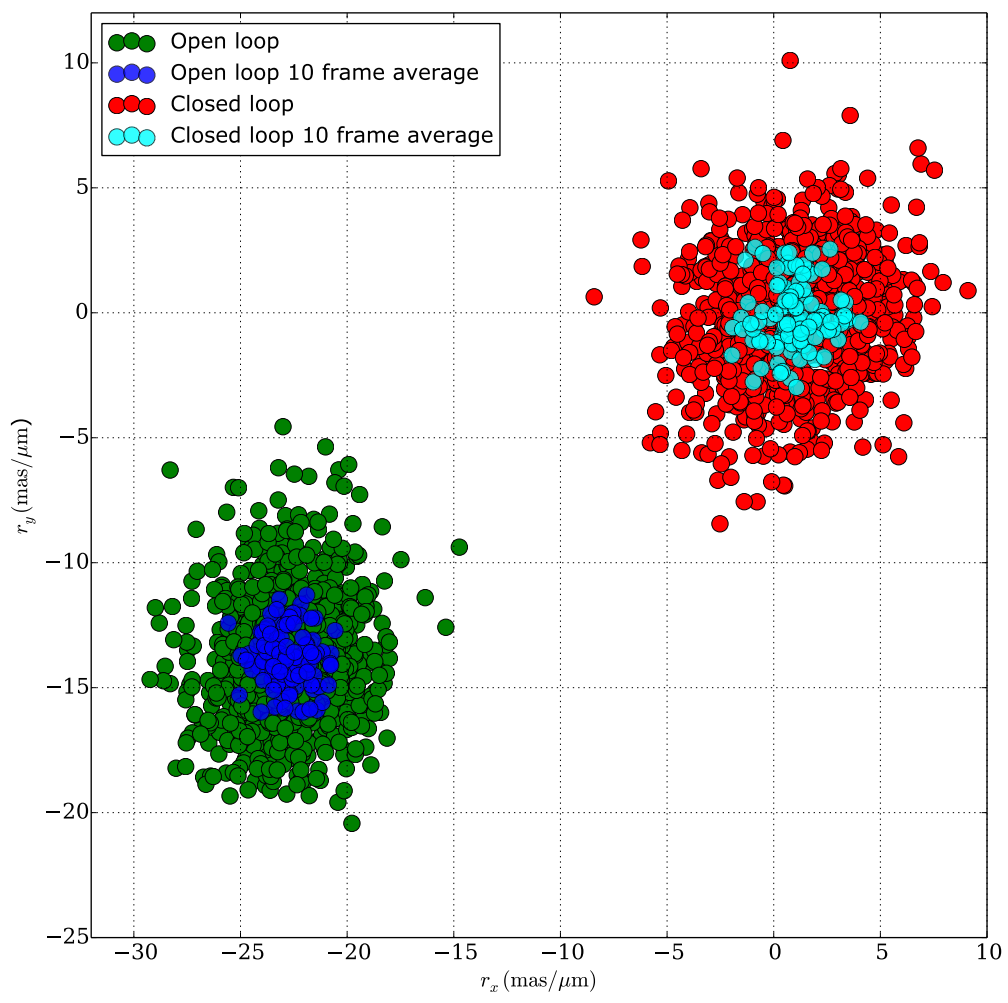

Figure 6. Scatter plot showing the effect binning and averaging for the on-sky measurement of the dispersion.

with a phase singularity in its center. Upon propagation from the focal plane to the pupil plane, azimuthal phase ramp sends the light outside the original pupil area, where it can be stopped by a Lyot stop. VVC is sensitive to the central obscuration, telescope support structures (spiders), low-order aberrations (tip-tilt, focus, etc) and bandwidth (chromaticity). ${ }^{9}$ For detailed theory of the VCC please see ${ }^{10}$

The presence of residual dispersion becomes a dominant noise source for small IWA coronagraphs once the other wavefront errors have been accounted for. To test the impact of closed-loop correction of the dispersion on the performance of small IWA vortex coronagraph, a high Strehl ratio (preferably > 90\%) and a fine correction of coronagraphic low-order modes are required. At the time of the test due to limitations in the SCExAO control software, Pyramid Wavefront Sensor (PyWFS) and Lyot Low Order Wavefront Sensor (LLOWFS) could not be employed simultaneously while using the satellite speckles for the dispersion measurement. To overcome this limitation, the following strategy was used:

1. The coronagraph flux leakage test was performed within a 10-minute window and it is assumed that the static atmospheric dispersion did not change considerably within that window.

2. Next, on-sky closed-loop correction of dispersion (including dispersion from the atmosphere and internaloptics) was performed. Then the loop was paused and satellite speckles were removed.

3. The PyWFS loop was closed to provide high Strehl ratio.

4. The LLOWFS loop was closed with vortex coronagraph to provide a fine correction of tip/tilt, focus and astigmatisms.

5. Coronagraphic data were collected using the previously closed-loop correction of dispersion.

6. Prism offsets used by closed-loop correction of the dispersion were removed. The ADC was reverted back to the look-up table based correction. 
(a)

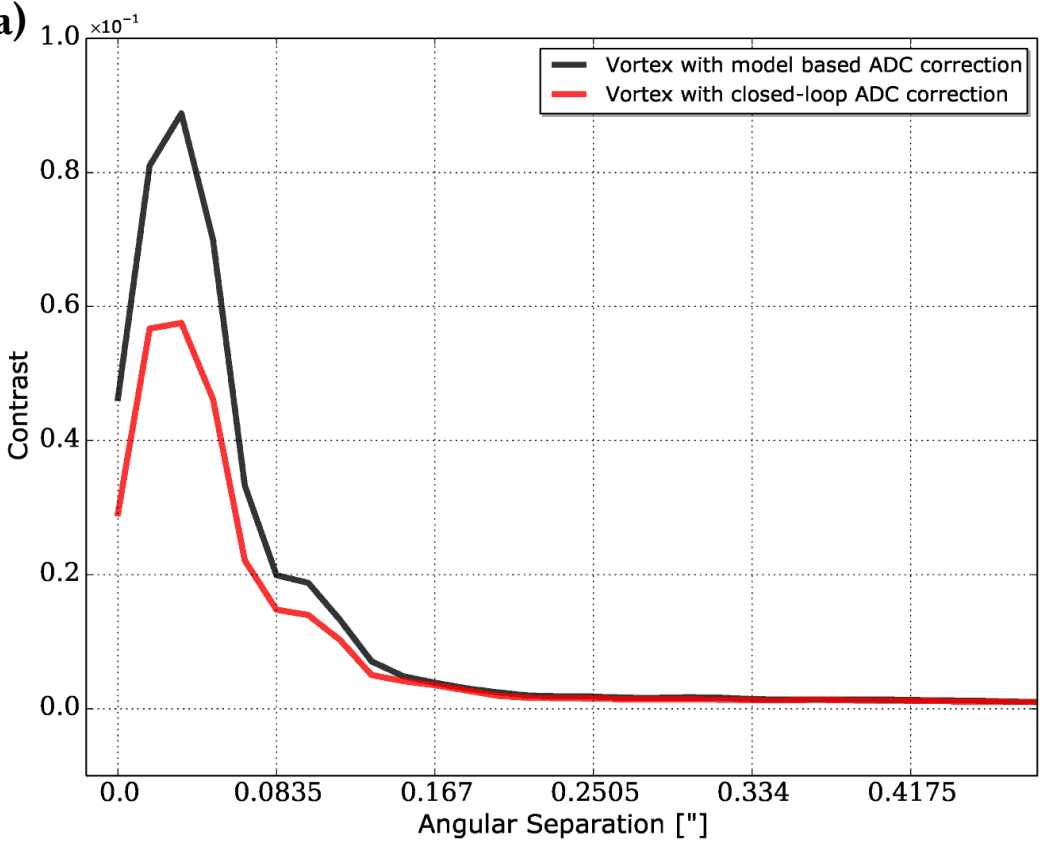

(b)

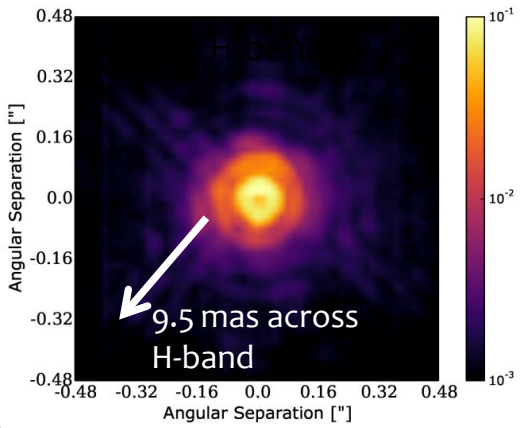

(c)

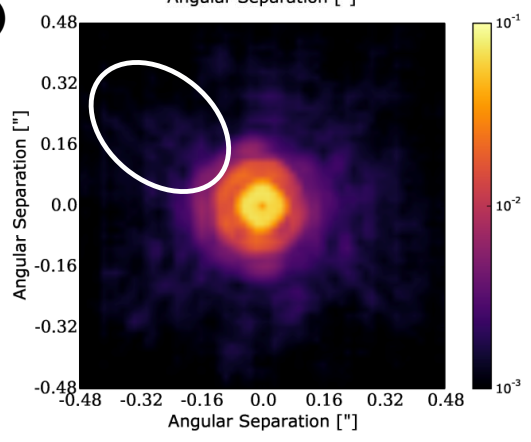

Figure 7. (a) Vortex coronagraph contrast curve as a function of angular separation for closed-loop and look-up table based ADC correction. (b) Vortex PSF with look-up table based ADC correction, having 9.5 mas of residual dispersion. (c) With closed-loop ADC correction.

7. Coronagraphic data were again collected for look-up table based correction of the dispersion.

Using the steps explained above, the on-sky data was collected on the target W Hya (spectral type M7, $\mathrm{V}-\mathrm{mag}=7.70, \mathrm{H}-\mathrm{mag}=-2.56)$ on SCExAO's engineering night of March $13^{t h}, 2017$. The telescope elevation during the data collection was $41^{\circ}$. The result of this on-sky experiment is shown in Figure 7. The data shown in the figure represents an average of 1000 co-added frames. The raw contrast curve (with no post-processing) for the VVC PSF as shown in Fig. 7 (a) was calculated by dividing maximum of the flux from a PSF without coronagraph. The raw contrast curve show in Figure. 7 (a) was generated by taking a radial average from center of the VVC PSF. The comparison between the raw contrast curve for look-up table based and closed-loop correction of the dispersion is shown in Fig. 7 (a). The figure shows an improvement in the raw contrast at small separations $<100$ mas, where the starlight leakages are the smallest for a closed-loop correction of the on-sky dispersion compared to a look-up table based correction. The improvement was marginal because at time of the test, we were dominated by low-order aberrations, especially tip-tilt variations. Figure 7 (b) shows an on-sky vortex PSF with the look-up table based ADC correction, the measured residual dispersion was 9.5 mas, direction is shown by an arrow. Figure 7 (c) shows a vortex PSF with closed-loop ADC correction. The diffraction features from spiders are more visible in Fig. 7 (b) compared to that of Fig. 7 (c), which clearly shows the leakage of light due to the presence residual dispersion.

\section{DISCUSSION}

In this work, we have demonstrated closed-loop measurement and correction of residual dispersion to a highprecision by applying fine corrections to the prisms of ADC, as an offset to the look-up table based correction presently used. In closed-loop we managed to achieve $<1$ mas of elongation in the PSF across H-band. We also show improvement in coronagraphic contrast with closed-loop correction of dispersion compared to the conventional look-up table based correction. The vortex coronagraph used for on-sky testing has an IWA of $1.5 \lambda / D$. The effect of dispersion will be even worse for $1 \lambda / D$ IWA coronagraphs. During on-sky data collection, the image quality was dominated by residual wavefront errors (providing a Strehl ratio of $60-80 \%$ in H-band), 
poor low-order correction and telescope vibration. However, we were still able to show the effect of residual dispersion on the performance of vortex coronagraph. The residual dispersion will be dominant source of noise once the other noise terms have been addressed. So the work presented here, will help to mitigate the effects of residual dispersion yielding in a better contrast for small IWA coronagraphs. The work presented here will pave the way to take spectra of Earth-like exoplanets and enable sub-milliarcsecond astrometry (to constrain the orbital parameters of exoplanets) in the era of ELTs.

This work can also be used as a diagnostic tool to measure the dispersion due to internal optics in the final science image and it can also be used to test and calibrate the look-up table based correction of ADCs. For future implementation of this method to perform a closed-loop correction of dispersion on ELTs. An error budget study of the temporal variation of dispersion due to atmospheric tip-tilt needs to be carried out for future ADC designs to address the dynamic component of the dispersion.

\section{ACKNOWLEDGMENT}

The development of SCExAO was supported by the JSPS (Grant-in-Aid for Research 23340051, 26220704, 23103002), the Astrobiology Center (ABC) of the National Institutes of Natural Sciences, Japan, the Mt Cuba Foundation and the directors contingency fund at Subaru Telescope. The authors wish to recognize and acknowledge the very significant cultural role and reverence that the summit of Maunakea has always had within the indigenous Hawaiian community. We are most fortunate to have the opportunity to conduct observations from this mountain.

\section{REFERENCES}

[1] Allen, C. V., [Astrophysical Quantities], The Athlone Press, London and Atlantic Highlands (1997 (third edition)).

[2] Wynne, C. G., "Correction of atmospheric dispersion in the infrared," mnras 282, 863-867 (oct 1996).

[3] Devaney, N., Goncharov, A. V., and Dainty, J. C., "Chromatic effects of the atmosphere on astronomical adaptive optics," Appl. Opt. 47, 1072-1081 (Mar 2008).

[4] Pathak, P., Guyon, O., Jovanovic, N., Lozi, J., Martinache, F., Minowa, Y., Kudo, T., Takami, H., Hayano, Y., and Narita, N., "A high-precision technique to correct for residual atmospheric dispersion in high-contrast imaging systems," Publications of the Astronomical Society of the Pacific 128(970), 124404 (2016).

[5] Pathak, P., Guyon, O., Jovanovic, N., Lozi, J., Martinache, F., Minowa, Y., Kudo, T., Takami, H., Hayano, Y., and Narita, N., "First on-sky closed loop measurement and correction of atmospheric dispersion," in [Adaptive Optics Systems V], Proc. SPIE 9909, 990956 (July 2016).

[6] Jovanovic, N., Martinache, F., Guyon, O., Clergeon, C., Singh, G., Kudo, T., Garrel, V., Newman, K., Doughty, D., Lozi, J., Males, J., Minowa, Y., Hayano, Y., Takato, N., Morino, J., Kuhn, J., Serabyn, E., Norris, B., Tuthill, P., Schworer, G., Stewart, P., Close, L., Huby, E., Perrin, G., Lacour, S., Gauchet, L., Vievard, S., Murakami, N., Oshiyama, F., Baba, N., Matsuo, T., Nishikawa, J., Tamura, M., Lai, O., Marchis, F., Duchene, G., Kotani, T., and Woillez, J., "The subaru coronagraphic extreme adaptive optics system: Enabling high-contrast imaging on solar-system scales," Publications of the Astronomical Society of the Pacific 127(955), 890 (2015).

[7] Minowa, Y., Hayano, Y., Oya, S., Watanabe, M., Hattori, M., Guyon, O., Egner, S., Saito, Y., Ito, M., Takami, H., Garrel, V., Colley, S., Golota, T., and Iye, M., "Performance of subaru adaptive optics system ao188," Proc. SPIE 7736(77363N), 77363N-7 (2010).

[8] Egner, S., Ikeda, Y., Watanabe, M., Hayano, Y., Golota, T., Hattori, M., Ito, M., Minowa, Y., Oya, S., Saito, Y., Takami, H., and Iye, M., "Atmospheric dispersion correction for the subaru ao system," Proc. SPIE 7736, 77364V-77364V-9 (2010).

[9] Mawet, D., Serabyn, E., Liewer, K., Hanot, C., McEldowney, S., Shemo, D., and O'Brien, N., "Optical vectorial vortex coronagraphs using liquid crystal polymers: theory, manufacturing and laboratory demonstration," Opt. Express 17, 1902-1918 (Feb 2009).

[10] Mawet, D., Riaud, P., Absil, O., and Surdej, J., "Annular groove phase mask coronagraph," The Astrophysical Journal 633(2), 1191 (2005). 